\title{
Solution-Based High-Density Arrays of Dielectric Microsphere Structures for Improved Crystal Quality of III-Nitride Layers on Si Substrates
}

\author{
Ho-Jun Lee, ${ }^{1}$ Kye-Jin Lee, ${ }^{2}$ Kwang-Yong Choi, ${ }^{2}$ Jung-Hyun Eum, ${ }^{2}$ Dong-Kun Lee, ${ }^{3}$ \\ Dong-Seon Lee, ${ }^{4}$ and Si-Young Bae ${ }^{1}$ \\ ${ }^{1}$ Department of Electrical Engineering and Computer Science, Nagoya University, Nagoya, Aichi 464-8603, Japan \\ ${ }^{2}$ HPC Advanced Development Team, LG Innotek, Paju, Gyeonggi-do 413-901, Republic of Korea \\ ${ }^{3}$ Wafer Characteristics Research Team, LG Siltron, Gumi, Gyeongsangbuk-do 730-724, Republic of Korea \\ ${ }^{4}$ School of Information and Communications, Gwangju Institute of Science and Technology (GIST), \\ Gwangju 500-712, Republic of Korea
}

Correspondence should be addressed to Si-Young Bae; siyoubae@gmail.com

Received 18 September 2015; Accepted 5 November 2015

Academic Editor: Jae-Woong Jeong

Copyright (C) 2015 Ho-Jun Lee et al. This is an open access article distributed under the Creative Commons Attribution License, which permits unrestricted use, distribution, and reproduction in any medium, provided the original work is properly cited.

\begin{abstract}
The recent development of dielectric microsphere lithography has been able to open up new means of performing simple and easy patterning on the semiconductor surfaces. Here, we report uniform and high-density arrays of microspheres using a solutionbased spin-coating method. The arrays of microspheres were used for etching mask to form the arrays of III-nitride microrods. By regrowing GaN layer on the microrod structures, high-quality GaN layer was achieved in terms of surface morphology as well as XRD characterization. To apply the advantages such as improved crystal quality and light extraction enhancement, light-emitting diodes (LEDs) were grown and then fabricated. The regrown LEDs with microspheres showed much improved optical output power and forward voltage characteristics in the same current injection. Therefore, we believe that this approach is quite useful for the development of high efficiency LEDs for future lighting.
\end{abstract}

\section{Introduction}

Colloidal particles have been widely used owing to their material/chemical/biological functionality [1]. Dimension of the colloidal particles can mainly be determined by the type of materials; it can thus be available for diverse applications such as slurries, clays, minerals, and aerosols [1]. The controlled size of the colloidal particles gives rise to special interests in the nanoscale patterning technology since they can provide a simple approach to form monodisperse masks [2]. In particular, the use of silica colloids is quite useful in inorganic solid-state lighting semiconductors since it not only gives realistic dimension of $10-1000 \mathrm{~nm}$, but also enables change of light management [1]. Moreover, the submicrometer-scale patterns can open up new opportunities for the semiconductor to be used for biomimetic applications [3].
One practical application of silica colloids can be to improve light extraction efficiency of light-emitting diodes (LEDs). To date, internal quantum efficiency (IQE) of LEDs is almost saturated $(>80 \%)$ owing to high-quality $\mathrm{GaN}$ epitaxial growth techniques $[4,5]$. Besides a design of multiple quantum wells (MQWs), many efforts in LEDs have been made to improve external quantum efficiency such as a reduction of polarization field effects and surface roughening $[6,7]$. In particular, the enhancement of light extraction efficiency (LEE) is of critical importance since the angle of light emission is limited by the large difference of refractive index between a semiconductor layer and air (or layer to layer) [8]. Hence, surface patterning and near field enhancement such as surface plasmon polariton (SPP) can be mostly highlighted for improving LEE [7, 9-11]. However, the fabrication cost to form a designed pattern on a surface or metal nanoparticles is 


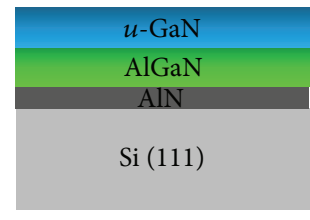

GaN epilayer growth

(a)

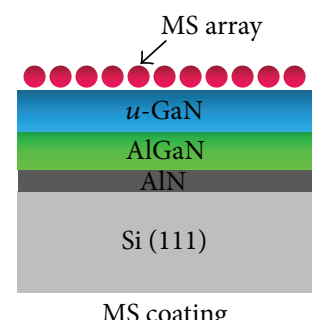

(b)

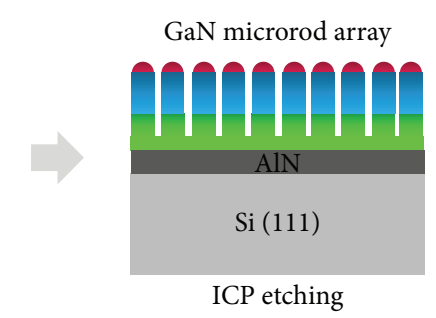

(c)

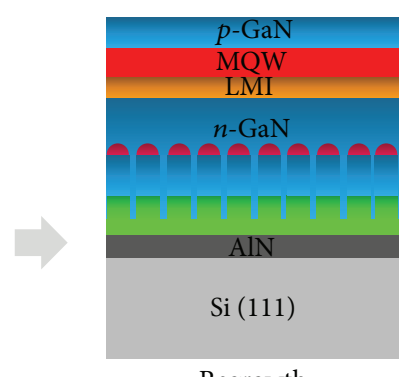

Regrowth

(d)

FIGURE 1: Schematic of the fabrication of LEDs with MS. (a) Epistructures, (b) MS coating, (c) dry etching, and (d) regrowth.

relatively expensive, making it undesirable regarding the cost reduction issue in the LED industry.

In this study, we report high-density and monodisperse arrays of dielectric microsphere using solution-based silica colloids. It is used as a two-dimensional (2D) etching mask to fabricate arrays of III-nitride microrods. By using a regrowth process in metalorganic chemical vapor deposition (MOCVD), we achieved much improved crystal quality of the III-nitride layer. Furthermore, LED chips with embedded microspheres and microrods were fabricated, showing dramatically improved light output power compared to the controlled LEDs. This method can not only provide costeffective fabrication compared with metal-used patterning, but also block the light absorption on Si substrates owing to the light reflection from the bottom side by inserting novel dielectric particles.

\section{Experimental Method}

Synthesis of silica particles was carried out by Stöber method. Arrays of dielectric microspheres (MS) were obtained by mixing silica particles in solution with deionized (DI) water, methanol, ammonia $\left(\mathrm{NH}_{3}\right)$, and diluted tetraethyl orthosilicate (TEOS). The uniformity of silica particles was controlled by sonication, and the diameter of silica particles became $\sim 1 \mu \mathrm{m}$. To generate a highly ordered and monodisperse layer, spin coating was performed at $600 \mathrm{rpm}$ for $1 \mathrm{~min}$. To prepare the $\mathrm{GaN}$ template on $\mathrm{Si}$ substrates, epilayers were grown on 2-inch Si (111) substrates by MOCVD (AIXTRON AIX 2000). Precursors of III and V sources were trimethylgallium (TMGa), trimethylaluminum (TMAl), trimethylindium (TMIn), and ammonia $\left(\mathrm{NH}_{3}\right)$. As dopants, disilane $\left(\mathrm{Si}_{2} \mathrm{H}_{6}\right)$ and cyclopentadienyl magnesium $\left(\mathrm{Cp}_{2} \mathrm{Mg}\right)$ were used to grow n-type and p-type GaN, respectively. Firstly, Si (111) substrates were heated at $1080^{\circ} \mathrm{C}$ in a $\mathrm{H}_{2}$ atmosphere for $10 \mathrm{~min}$ to remove native oxide on the surface. To avoid surface damage due to melt-back etching, TMAl source was first opened for $10 \mathrm{~s}$ before the $\mathrm{NH}_{3}$ injection. A $60 \mathrm{~nm}$ thick high-temperature AlN (HT-AlN) buffer layer was grown at $1020^{\circ} \mathrm{C}$ with $\mathrm{Al}$ nucleation process. Subsequently, a threestep AlGaN buffer layer with a thickness of $\sim 600 \mathrm{~nm}$ was grown, where $\mathrm{Al}$ compositions were composed of $76 \%$, $54 \%$, and $23 \%$ from bottom to top layer, respectively. After growing a $500 \mathrm{~nm}$ thick $u$-GaN layer at $1045^{\circ} \mathrm{C}$, a $15 \mathrm{~nm}$ thick low-temperature AlN (LT-AlN) layer was grown at $820^{\circ} \mathrm{C}$ to reduce the threading dislocation originated from initial growth procedures. A $400 \mathrm{~nm}$ thick $u$-GaN was additionally grown at $1045^{\circ} \mathrm{C}$, after which we could obtain a GaN template to disperse MS particles.

After forming spin-coated MS mask arrays, the samples were etched by inductively coupled plasma (ICP) etcher. The dry etching was performed at 5 mTorr with $\mathrm{Cl}_{2}$ $(30 \mathrm{sccm}) / \mathrm{BCl}_{3}(28 \mathrm{sccm})$ gas mixtures. To proceed with a regrowth process, a $1.5 \mu \mathrm{m} n$-GaN (Si doping concentration is $5 \times 10^{18} \mathrm{~cm}^{-3}$ ) was grown to merge the empty gaps of the microrod arrays. Then, $30 \mathrm{~nm} \mathrm{In}_{0.05} \mathrm{Ga}_{0.95} \mathrm{~N} / \mathrm{GaN}$ superlattices (SLs) for a lattice-matched layer (LMI) and seven pairs of multiple quantum wells (MQWs) were grown. Here, a $20 \mathrm{~nm} p-\mathrm{Al}_{0.21} \mathrm{Ga}_{0.79} \mathrm{~N}$ layer was also grown, which plays a role in an electron blocking layer (EBL). Finally, a $100 \mathrm{~nm}$ $p$-GaN layer and a $20 \mathrm{~nm} p+\mathrm{GaN}$ capping layer were grown at the same growth temperature of $960^{\circ} \mathrm{C}$, where p-type doping concentration was maintained at $\sim 1 \times 10^{20} \mathrm{~cm}^{-3}$. Figure 1 shows the schematic of the LEDs with MS in detail. The surface morphology of grown epilayers was observed using scanning electron microscope (SEM, Hitachi S-4700) and optical microscope (OM, Olympus). Crystal quality of the grown $\mathrm{GaN}$ epilayer was characterized using X-ray diffraction (XRD, Philips X'Pert) equipped with a hybrid monochromator (2-bounce) in the incident beam and a channel-cut Ge (220) diffraction beam. Electrical and optical properties of fabricated devices were characterized by an LED chip measurement system. The size of a single chip was $500 \times$ $500 \mu \mathrm{m}^{2}$.

\section{Results and Discussion}

Prior to proceeding with the regrowth in MOCVD, a GaN template was prepared for dispersing MS particles, as shown in the schematic in Figure 2(a). It consists of a $60 \mathrm{~nm}$ HTAlN layer, a $600 \mathrm{~nm}$ graded AlGaN layer, a $500 \mathrm{~nm}$ undoped GaN layer, a $15 \mathrm{~nm}$ LT-AlN layer, and a $400 \mathrm{~nm}$ undoped GaN layer. Here, we used 3-step AlGaN layers aiming to release the biaxial strain from GaN/Si interface, reducing cracks during cooling process of epitaxy [12]. The HT-AlN layer was grown to avoid melt-back etching from the chemical reaction 


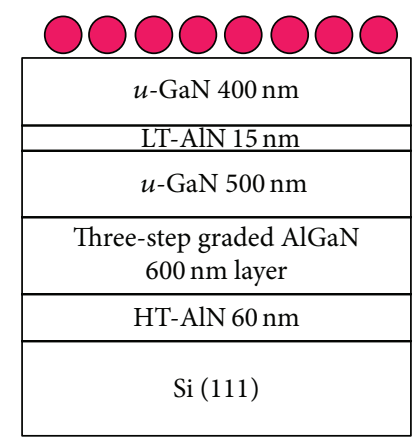

(a)

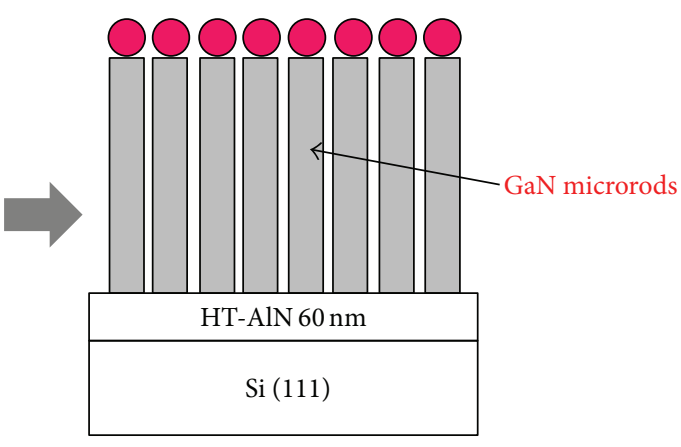

(b)

FIGURE 2: Schematic of (a) grown epitaxial GaN structures on Si (111) substrates with dispersed MS monolayer and (b) microrod arrays after ICP etching.

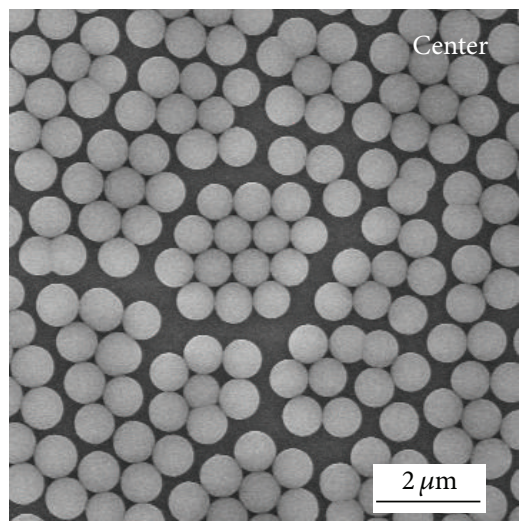

(a)

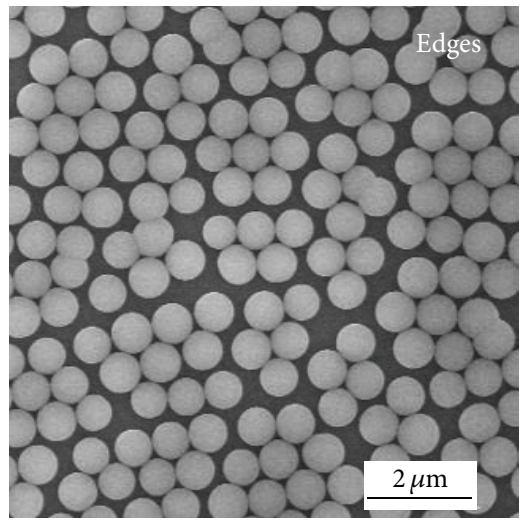

(b)

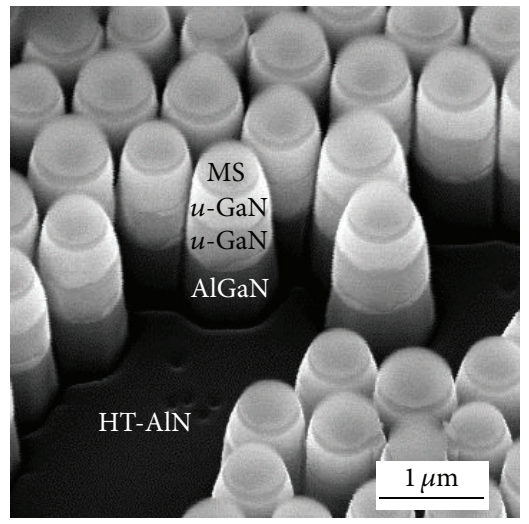

(c)

FIGURE 3: Top-view SEM images of dispersed MS structures on (a) center and (b) edge regions. (c) Tilt-view SEM image of microrod structures after ICP etching.

between $\mathrm{Si}$ and $\mathrm{Ga}$ atoms, and an LT-AlN layer was inserted to reduce threading dislocation initiated from $\mathrm{Si}$ substrates [13]. Then, a monolayer with a high density of MS arrays was dispersed on the planar GaN template (Figure 2(a)). By performing the ICP etching process with $\mathrm{Cl}_{2}$-based gas mixtures, arrays of III-nitride microrods could be obtained, as shown in the schematic in Figure 2(b). To avoid the meltback etching in high growth temperatures, dry etching was stopped at the HT-AlN layer. Figure 3 shows the SEM images for the fabricated microstructures. Figures 3(a) and 3(b) display the top-view SEM images of dispersed microspheres at center and edge regions, respectively. For further feasible applications of the spin-coated microstructures, the MS uniformity is of importance. It requires not only to be formed as a monolayer but also to maintain high density. If these two requirements are not satisfied, the regrown structures could not keep the smooth surface morphology due to large difference of coalescence distance [14]. The surface coverages of coated MS were $\sim 80 \%$ and $\sim 83 \%$ for center and edge regions of a 2-inch wafer, respectively. Moreover, the surface was almost covered by arrays of a monolayer, except for a few regions overlapped by the MS. Figure 3(c) shows a tilt-view SEM image of arrays of III-nitride microrods after the dry etching process. Each region for single microrods was clearly distinguished since the AlGaN alloys showed a distinct brightness compared with that of the GaN layer. The MS remained on the top of the microrods, indicating a chemical stability for dry etching process.

As the next step, an $n$-GaN regrowth was carried out in MOCVD. For the full coalescence, we grew $n-\mathrm{GaN}$ with thickness of $\sim 1.5 \mu \mathrm{m}$. To fairly compare the regrown $\mathrm{GaN}$ layers, a planar $\mathrm{GaN}$ layer was also grown as a controlled sample. By performing an XRD $\omega$ scan for two samples, we could investigate crystal quality of epitaxial layers. In (002) reflection, the full width of half maximum (FWHM) of regrown GaN layers with MS was 394 arcsec, while that of the controlled sample without MS was 640 arcsec, as shown in Figure 4(a). Furthermore, the FWHM was decreased from 925 to 478 arcsec by applying the regrowth over the microrod structures in (102) reflection. The pits of the GaN surface were also dramatically reduced and surface smoothness with terrace-like atomic features was observed (not shown). This indicates the enhanced crystal quality, as observed from XRD characterization.

Figure 5 shows the SEM images of regrown $\mathrm{GaN}$ as growth time increases from 5 to $20 \mathrm{~min}$. At the beginning, 

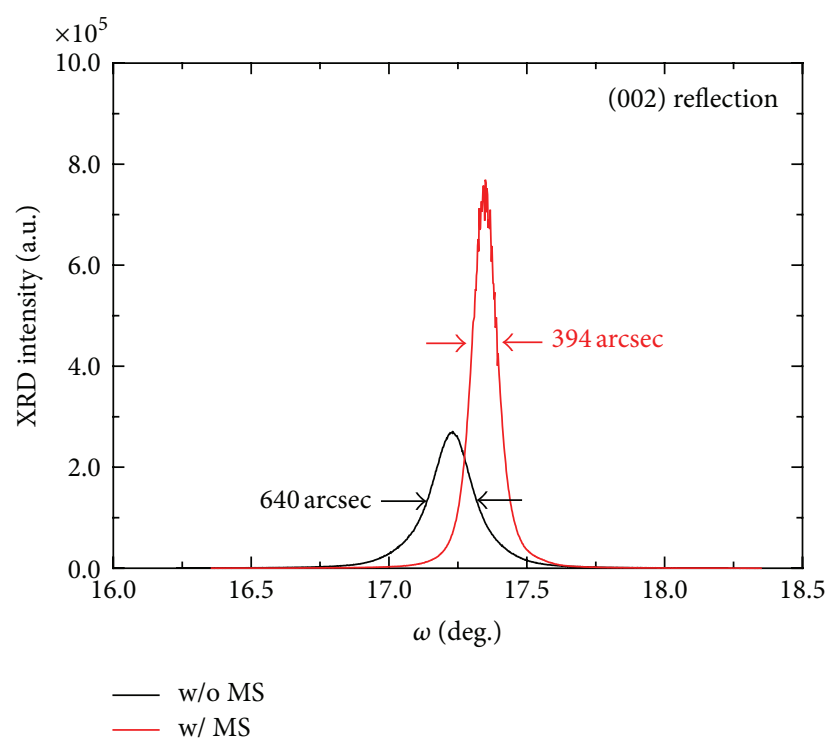



(b)

FIGURE 4: FWHM of XRD $\omega$ scan for GaN layers with MS and without MS structures in (a) (002) and (b) (102) reflections.

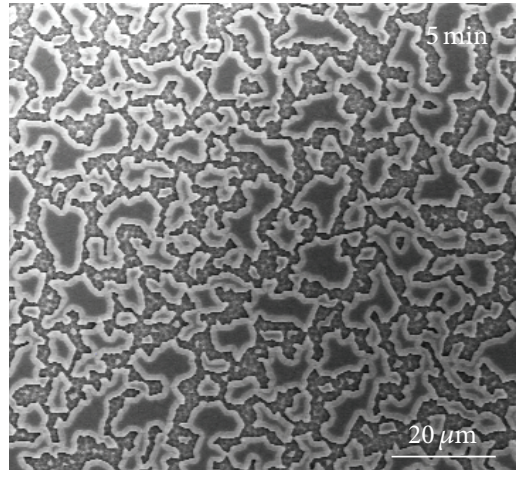

(a)

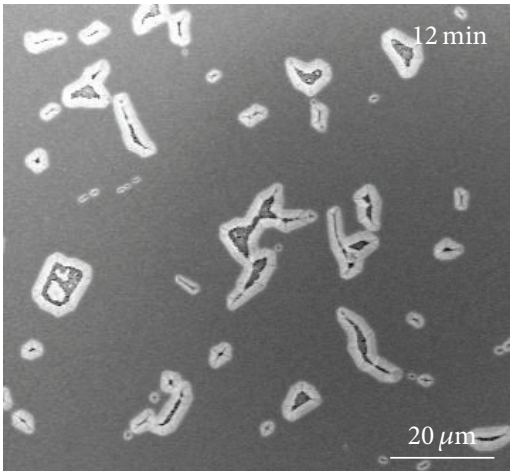

(b)



(c)

FIGURE 5: SEM images of regrown GaN structures at (a) $5 \mathrm{~min}$, (b) $12 \mathrm{~min}$, and (c) $20 \mathrm{~min}$.

the three-dimensional (3D) island-like morphology was observed where the 3D layers were randomly distributed over the whole surface (Figure 5(a)). Then, 2D dominant morphology was shown except for partially empty regions (Figure 5(b)). Finally, smooth 2D layers were grown in $20 \mathrm{~min}$, and after that these 2D layers were thickened by increasing the growth time further (Figure 5(c)). During the coalescence process, several factors can contribute to the improvement of crystal quality from MS structures: dislocation bending, defect blocking, strain suppression, and so forth [15-18]. As evident from the improved FWHM in XRD measurement, we believe that not only dislocations from the templates were reduced but also strain above the MS was relaxed during the microrod etching and coalescence process $[18,19]$.

We further grew the LED structures on both the planar and regrown layers to investigate the effect of electroluminescence by using microrod structures. Figures 6(a) and 6(b) show the schematic of grown structures. The LED structures consist of $30 \mathrm{~nm} \operatorname{In}_{0.05} \mathrm{Ga}_{0.95} \mathrm{~N} / \mathrm{GaN}$ SLs, seven multiple quantum wells (InGaN/GaN $=2.5 \mathrm{~nm} / 12 \mathrm{~nm}$ ), $20 \mathrm{~nm} p$ AlGaN layer, $100 \mathrm{~nm} p$-GaN layer, and $20 \mathrm{~nm} p+\mathrm{GaN}$ layer. Here, the LMI was inserted for releasing the biaxial strain at InGaN/GaN interfaces of MQWs; it is thereby able to increase indium incorporation as well as internal quantum efficiency [20]. For fabricating LED chips, conventional photolithography and electrode formation were formed on both grown epitaxial structures. Figure 7 shows the typical spectra of both LED structures at the current injection of $20 \mathrm{~mA}$. The EL intensity of LEDs with MS was significantly improved compared to that of LEDs without MS. The details of device performance are described in Table 1. By measuring the optical output power in the integrating sphere, it was improved from 3.08 to $4.11 \mathrm{~mW}$, a $33 \%$ increase. Several factors could affect this optical power improvement, such as enhanced light extraction, crystal quality, and indium 


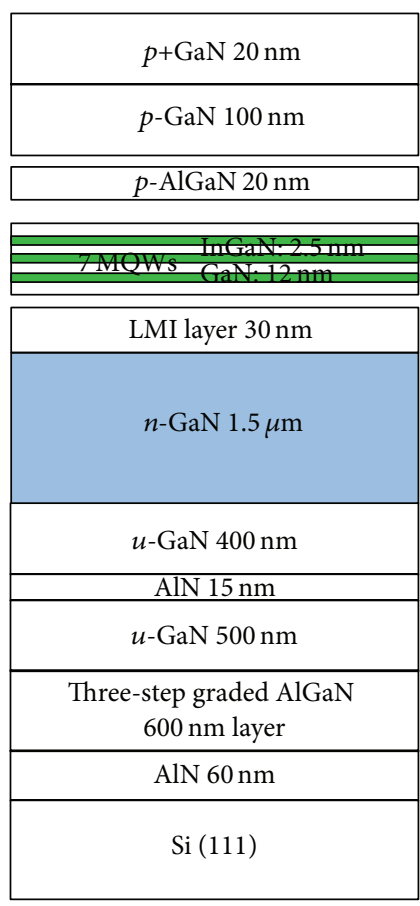

(a)

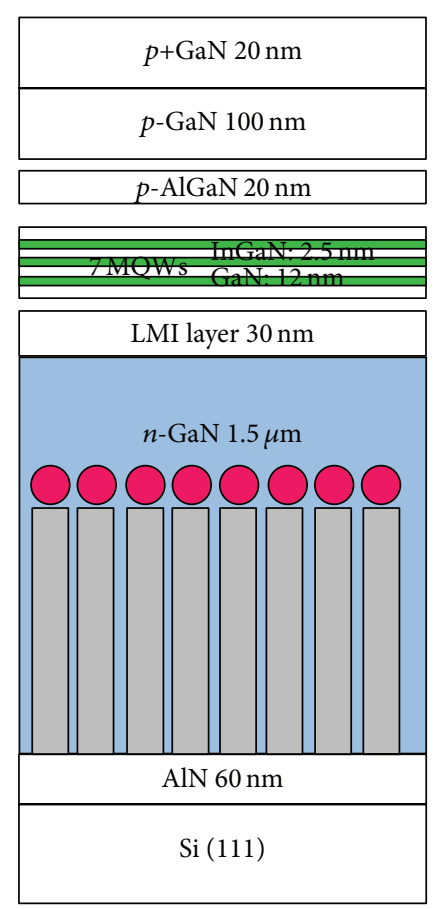

(b)

FIGURE 6: Schematic of (a) planar LED structures on Si (111) substrates without MS and (b) regrown LED structures with MS.



FIGURE 7: EL spectra of fabricated LEDs at current injection of $20 \mathrm{~mA}$ with and without MS structures.

homogeneity $[7,9,21]$. In particular, the regrown $\mathrm{GaN}$ layer includes embedded MS structures for enhanced light extraction. We can expect more increased light scattering from this structure owing to the largely different refractive index $(n)$ between GaN ( $n=\sim 2.4$ at a wavelength of $555 \mathrm{~nm}$ ) and MS $(n=\sim 1.5)$ [22-24]. This can be highly advantageous for $\mathrm{GaN}$ on $\mathrm{Si}$ epitaxy since the strong light absorption in Si can be reduced from this light management. In addition, the electrical properties of LEDs can be critically affected by
TABLE 1: Summary of device performance with peak wavelength $\left(\lambda_{\text {peak }}\right)$ at $20 \mathrm{~mA}$, forward voltage $\left(V_{f}\right)$, and optical output power $\left(P_{o}\right)$.

\begin{tabular}{lccc}
\hline Samples & $\lambda_{\text {peak }}(\mathrm{nm}) @ 20 \mathrm{~mA}$ & $V_{f}(\mathrm{~V})$ & $P_{o}(\mathrm{~mW})$ \\
\hline LED w/o MS & 467.0 & 3.25 & 3.08 \\
LED w/ MS & 463.0 & 3.06 & 4.11 \\
\hline
\end{tabular}

a crystal quality of GaN layer on Si substrates [25]. Hence, the reduced forward voltage from 3.25 to $3.06 \mathrm{~V}$ can be attributed to the improved crystal quality, as evident from the FWHM of XRD in Figure 4. Even though the same growth condition was used for both LED samples, slight difference of peak wavelength might be due to the run-to-run deviation as well as spatial deviation.

In terms of prospective view for this method, we think that the density of MS (i.e., surface coverage of MS) is a very crucial parameter for LED performance. In brief, toohigh a density of MS (e.g., >95\%) is not useful for this regrowth approach since the fully covered MS layer does not provide enough room for a proper regrowth. In contrast, too-low a density of MS (e.g., <30\%) cannot contribute significantly to the improvement of crystal quality as well as light extraction of LEDs because the regrown layers seemed to be $2 \mathrm{D}$ without additional scattering structures. Figures 8 (a) and 8(b) show the representative SEM images for low density $(d=\sim 300 \mathrm{~nm})$ and high density $(d=\sim 800 \mathrm{~nm})$, respectively, where $d$ indicates a diameter of MS. In our case, the density of MS was somewhat increased by increasing the diameter of MS (Figure 8(c)). The highest density of MS was 


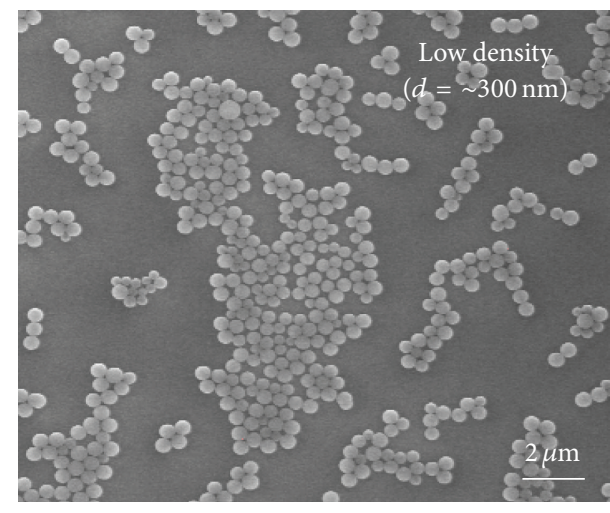

(a)

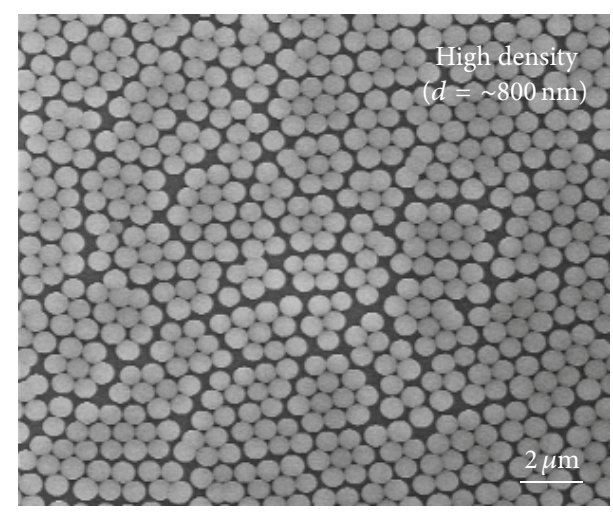

(b)

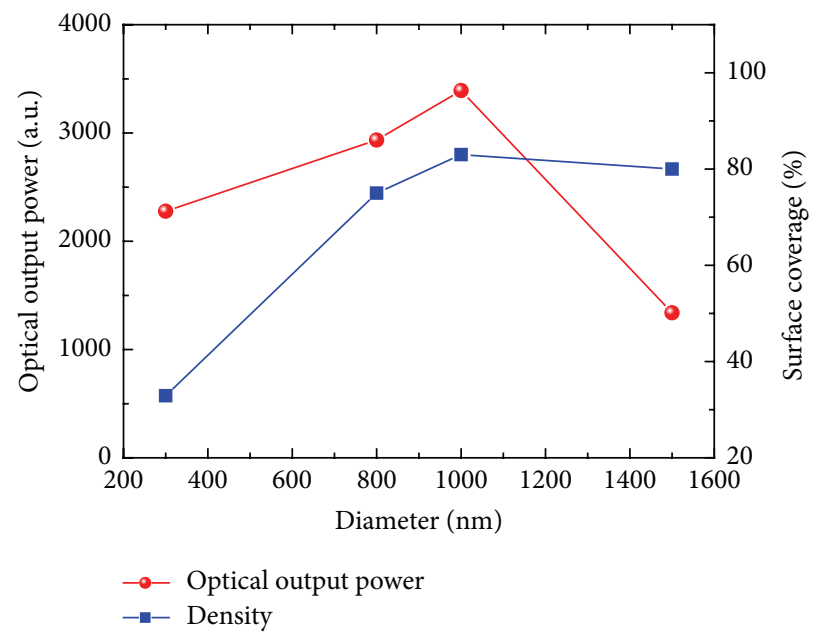

(c)

FIGURE 8: SEM images of (a) low density of MS with a diameter of $\sim 300 \mathrm{~nm}$ and (b) high density of MS with a diameter of $\sim 800 \mathrm{~nm}$. (c) Optical output power of LEDs and density of MS as a function of the diameter.

acquired at a diameter of $1000 \mathrm{~nm}$. We believe that the slight decrease of the density on the diameter of $1500 \mathrm{~nm}$ compared to the MS with the diameter of $\sim 800 \mathrm{~nm}$ is attributed to the reduced packing ratio due to the larger size of MS. Figure 8(c) also shows the optical output power of LEDs by applying different diameters of MS. Considerable improvement of the optical output power of MS was observed until the diameter became $\sim 1000 \mathrm{~nm}$. The exceptional decrease for the MS with a diameter of $1500 \mathrm{~nm}$, despite the high density $(\sim 80 \%)$, is due to poor coalescence during the regrowth. Note that the diameter of $\sim 1500 \mathrm{~nm}$ is quite similar to the total regrowth thickness of $n$-GaN. To merge the whole surface, at least, the longer growth time is required under the same growth condition. Therefore, we could achieve the highest optical output power of LEDs with the moderate density of MS ( $\sim 83 \%)$ and full coalescence of GaN over the whole surface.

\section{Conclusion}

In this paper, we reported improved quality of III-nitride epilayers on Si substrates using regrowth on microrod arrays. To demonstrate the microrod structures, MS arrays were uniformly dispersed on the surface by controlling the synthesis condition, thereby being used for masks. Depending on the density of MS arrays, the density of microrod arrays was considerably affected since we used a top-down approach to form the rod structure. From the XRD analysis, the FWHM of regrown $\mathrm{GaN}$ was dramatically reduced, indicating high crystal quality. The fabricated LEDs not only enhanced optical output power by $33 \%$, but also reduced forward voltage characteristics at the same current injection. Therefore, we believe that this method can contribute to the improvement of the electrical and optical properties of LEDs for future lighting.

\section{Conflict of Interests}

The authors declare that there is no conflict of interests regarding the publication of this paper.

\section{Acknowledgment}

This research was supported by the Core Technology Development Program for Next-Generation Energy of Research Institute for Solar and Sustainable Energies (RISE). 


\section{References}

[1] Y. Xia, B. Gates, Y. Yin, and Y. Lu, "Monodispersed colloidal spheres: old materials with new applications," Advanced Materials, vol. 12, no. 10, pp. 693-713, 2000.

[2] J. C. Hulteen and R. P. Van Duyne, "Nanosphere lithography: a materials general fabrication process for periodic particle array surfaces," Journal of Vacuum Science and Technology A: Vacuum, Surfaces and Films, vol. 13, no. 3, pp. 1553-1558, 1995.

[3] J. N. Cha, G. D. Stucky, D. E. Morse, and T. J. Deming, "Biomimetic synthesis of ordered silica structures mediated by block copolypeptides," Nature, vol. 403, no. 6767, pp. 289-292, 2000.

[4] S. P. DenBaars, D. Feezell, K. Kelchner et al., "Development of gallium-nitride-based light-emitting diodes (LEDs) and laser diodes for energy-efficient lighting and displays," Acta Materialia, vol. 61, no. 3, pp. 945-951, 2013.

[5] H. Zhao, G. Liu, J. Zhang, J. D. Poplawsky, V. Dierolf, and N. Tansu, "Approaches for high internal quantum efficiency green InGaN light-emitting diodes with large overlap quantum wells," Optics Express, vol. 19, no. 14, pp. A991-A1007, 2011.

[6] M.-H. Kim, M. F. Schubert, Q. Dai et al., "Origin of efficiency droop in GaN-based light-emitting diodes," Applied Physics Letters, vol. 91, no. 18, Article ID 183507, 2007.

[7] T. Fujii, Y. Gao, R. Sharma, E. L. Hu, S. P. DenBaars, and S. Nakamura, "Increase in the extraction efficiency of GaN-based light-emitting diodes via surface roughening," Applied Physics Letters, vol. 84, no. 6, pp. 855-857, 2004.

[8] E. F. Schubert, Light-Emitting Diodes, E. Fred Schubert, 2nd edition, 2006.

[9] B. Sun, L. Zhao, T. Wei et al., "Light extraction enhancement of bulk GaN light-emitting diode with hemisphere-cones-hybrid surface," Optics Express, vol. 20, no. 17, pp. 18537-18544, 2012.

[10] S. X. Jin, J. Li, J. Z. Li, J. Y. Lin, and H. X. Jiang, "GaN microdisk light emitting diodes," Applied Physics Letters, vol. 76, no. 5, pp. 631-633, 2000.

[11] I. Gontijo, M. Boroditsky, E. Yablonovitch, S. Keller, U. K. Mishra, and S. P. DenBaars, "Coupling of InGaN quantum-well photoluminescence to silver surface plasmons," Physical Review B-Condensed Matter and Materials Physics, vol. 60, no. 16, pp. 11564-11567, 1999.

[12] A. Able, W. Wegscheider, K. Engl, and J. Zweck, "Growth of crack-free GaN on $\mathrm{Si}\left(\begin{array}{lll}1 & 1 & 1\end{array}\right)$ with graded AlGaN buffer layers," Journal of Crystal Growth, vol. 276, no. 3-4, pp. 415-418, 2005.

[13] D. Zhu, D. J. Wallis, and C. J. Humphreys, "Prospects of IIInitride optoelectronics grown on Si," Reports on Progress in Physics, vol. 76, no. 10, Article ID 106501, 2013.

[14] K. Hiramatsu, K. Nishiyama, A. Motogaito, H. Miyake, Y. Iyechika, and T. Maeda, "Recent progress in selective area growth and epitaxial lateral overgrowth of III-nitrides: Effects of reactor pressure in MOVPE growth," Physica Status Solidi A: Applied Research, vol. 176, no. 1, pp. 535-543, 1999.

[15] C.-Y. Hsieh, B.-W. Lin, H.-J. Cho, B.-M. Wang, and Y. C. Sermon $\mathrm{Wu}$, "Investigation of GaN films grown on liquid-phase deposited $\mathrm{SiO}_{2}$ nanopatterned sapphire substrates," ECS Journal of Solid State Science and Technology, vol. 1, no. 2, pp. Q35-Q37, 2012.

[16] K. Ueda, Y. Tsuchida, N. Hagura, F. Iskandar, K. Okuyama, and Y. Endo, "High performance of GaN thin films grown on sapphire substrates coated with a silica-submicron-sphere monolayer film," Applied Physics Letters, vol. 92, no. 10, Article ID 101101, 2008.
[17] R. Colby, Z. Liang, I. H. Wildeson et al., "Dislocation filtering in GaN nanostructures," Nano Letters, vol. 10, no. 5, pp. 1568-1573, 2010.

[18] J. Kim, H. Woo, K. Joo et al., "Less strained and more efficient GaN light-emitting diodes with embedded silica hollow nanospheres," Scientific Reports, vol. 3, article 3201, 2013.

[19] S.-Y. Bae, D.-J. Kong, J.-Y. Lee, D.-J. Seo, and D.-S. Lee, "Sizecontrolled InGaN/GaN nanorod array fabrication and optical characterization," Optics Express, vol. 21, no. 14, pp. 1685416862, 2013.

[20] E. Feltin, B. Beaumont, M. Laügt et al., "Stress control in GaN grown on silicon (111) by metalorganic vapor phase epitaxy," Applied Physics Letters, vol. 79, no. 20, pp. 3230-3232, 2001.

[21] C. Huh, W. J. Schaff, L. F. Eastman, and S.-J. Park, “Temperature dependence of performance of InGaN/GaN MQW LEDs with different indium compositions," IEEE Electron Device Letters, vol. 25, no. 2, pp. 61-63, 2004.

[22] X.-H. Li, R. Song, Y.-K. Ee, P. Kumnorkaew, J. F. Gilchrist, and N. Tansu, "Light extraction efficiency and radiation patterns of III-nitride light-emitting diodes with colloidal microlens arrays with various aspect ratios," IEEE Photonics Journal, vol. 3, no. 3, pp. 489-499, 2011.

[23] C.-C. Chen, C.-Y. Yap, W.-Y. Hsu et al., "Crystal quality and light output power of GaN-based LEDs grown on nanoporous patterned sapphire substrate," ECS Solid State Letters, vol. 3, no. 9, pp. R45-R47, 2014.

[24] D.-W. Jeon, L.-W. Jang, H.-S. Cho et al., "Enhanced optical output performance in InGaN/GaN light-emitting diode embedded with $\mathrm{SiO}_{2}$ nanoparticles," Optics Express, vol. 22, no. 18, pp. 21454-21459, 2014.

[25] J. K. Sheu, J. M. Tsai, S. C. Shei et al., "Low-operation voltage of InGaN/GaN light-emitting diodes with Si-doped $\operatorname{In}_{0.3} \mathrm{Ga}_{0.7} \mathrm{~N} /$ GaN short-period superlattice tunnelling contact layer," IEEE Electron Device Letters, vol. 22, no. 10, pp. 460-462, 2001. 

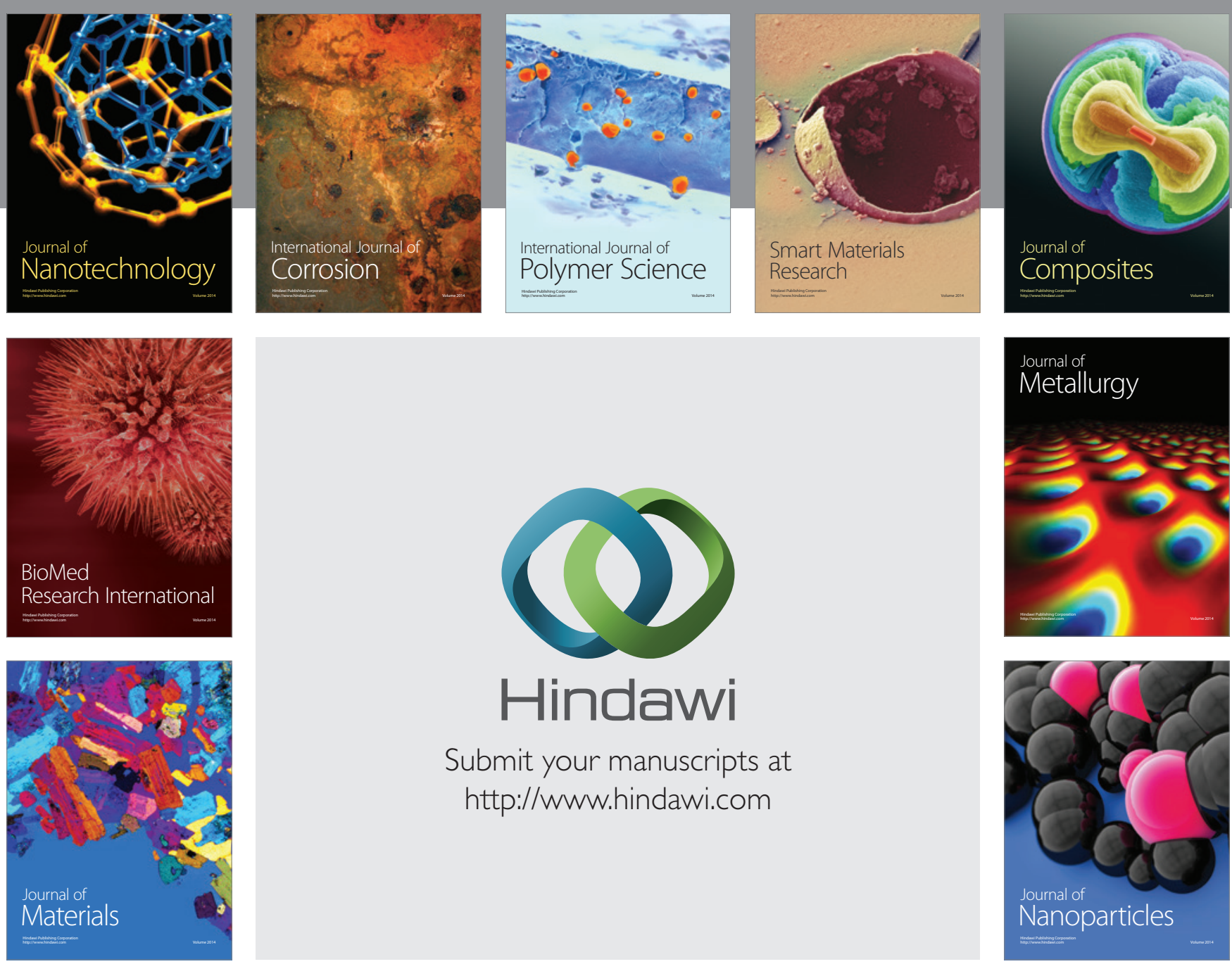

Submit your manuscripts at http://www.hindawi.com
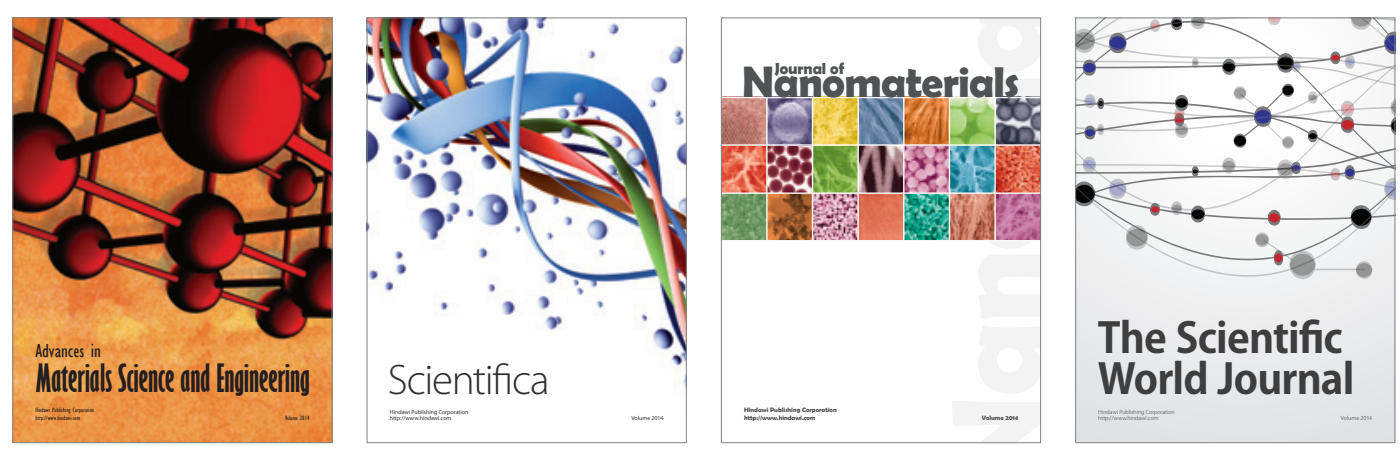

\section{The Scientific World Journal}
